\title{
Recent measurement of the Telescope Array energy spectrum and observation of the shoulder feature in the Northern Hemisphere
}

\section{Ivanov, ${ }^{a, *}$ D. Bergman, ${ }^{a}$ G. Furlich, ${ }^{a}$ R. Gonzalez, ${ }^{a}$ G. B. Thomson ${ }^{a}$ and Y.} Tsunesada $^{b}$ on behalf of the Telescope Array Collaboration

(a complete list of authors can be found at the end of the proceedings)

${ }^{a}$ University of Utah, Department of Physics \& Astronomy and High Energy Astrophysics Institute, Salt Lake City, Utah, USA

${ }^{b}$ Graduate School of Science, Osaka City University, Osaka, Osaka, Japan

E-mail: dmiivanov@gmail.com

The Telescope Array (TA) is a hybrid cosmic ray detector deployed in 2007 in Millard County, Utah, USA, which consists of a surface detector of 507 plastic scintillation counters spanning a $700 \mathrm{~km}^{2}$ area on the ground that is overlooked by three fluorescence detector stations. The High Resolution Fly's Eye (HiRes) experiment is a predecessor of TA, which consisted of two fluorescence detector stations operating from 1997 until 2006 from Dugway Proving Ground, Utah, USA, and which was the the first cosmic ray experiment with sufficient resolution and exposure to successfully observe the Greisen-Zatsepin-Kuzmin (GZK) suppression at $10^{19.75} \mathrm{eV}$. In this work, we present an updated TA energy spectrum result and a joint fit of independent spectrum measurements by the TA surface detector, TA fluorescence detector, and HiRes fluorescence detector to a broken power law function, which exhibits the ankle, GZK suppression, and the new shoulder feature initially seen by the Pierre Auger Observatory in the Southern Hemisphere. HiRes and TA observe the shoulder feature in the Northern Hemisphere at $10^{19.25} \mathrm{eV}$, with a statistical significance of 5.3 standard deviations.

$37^{\text {th }}$ International Cosmic Ray Conference (ICRC 2021)

July 12th - 23rd, 2021

Online - Berlin, Germany

\footnotetext{
${ }^{*}$ Presenter
} 


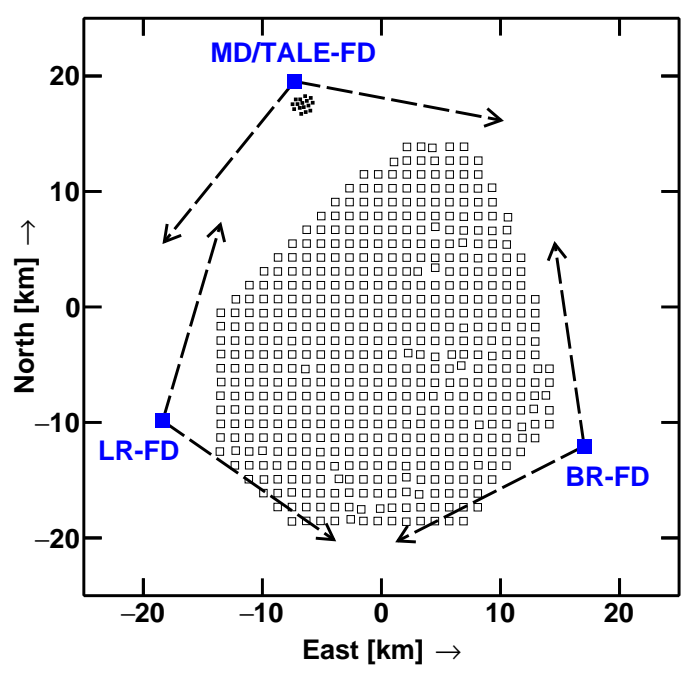

Figure 1: Layout of the Telescope Array (TA) detector. Open squares are the main TA surface detector (SD) counters, small filled squares are the TA Low Energy Extension (TALE) SD counters, and the large boxes show the TA fluorescence detector (FD) stations Black Rock Mesa (BR), Long Ridge (LR), and Middle Drum (MD). Arrows show the FD viewing ranges.

\section{Introduction}

The Telescope Array (TA) is a hybrid detector that measures cosmic rays mostly in the Northern Hemisphere. TA is located in Millard County, UT, USA and it is collecting data since March 2007. The main TA consists of a $\sim 700 \mathrm{~km}^{2}$ surface detector (SD) of 507 plastic scintillation counters [1] that is overlooked by three fluorescence detector (FD) stations [2, 3], as depicted in Figure 1.

The Telescope Array Black Rock (BR) and Long Ridge (LR) fluorescence detectors (FD) consist of 10 telescopes each, which are viewing 3 to $33^{\circ}$ elevations and a $2 \times 108^{\circ}$ azimuth range. The TA Middle Drum (MD) site consists of 14 telescopes that are covering 3 to $31^{\circ}$ elevations and an azimuth range of $112^{\circ}$. The Telescope Array Low Energy Extension (TALE) is an additional hybrid detector that extends the TA energy range down to $10^{15.4} \mathrm{eV}$. TALE consist of 10 HiRes II - type [4] fluorescence telescopes added at the TA MD site to cover $31^{\circ}$ to $57^{\circ}$ elevations and to overlook an infill array of 103 plastic scintillation counters.

The High Resolution Fly's Eye (HiRes) experiment, [4], was built on Dugway Proving Ground, UT, and was collecting data from 1997 to 2006 . HiRes had two fluorescence detectors, HiRes I and HiRes II, separated by $13 \mathrm{~km}$. HiRes experiment viewed 3 to $31^{\circ}$ in elevation and about $360^{\circ}$ in azimuth.

\section{TA Spectrum and the shoulder feature}

Figure 2 shows the Telescope Array (TA) combined energy spectrum produced using the 22 months TA Low Energy Extension (TALE) [5] measurement below $10^{18.2} \mathrm{eV}$ and an 11 year TA surface detector spectrum (SD) [7] above $10^{18.2} \mathrm{eV}$. 


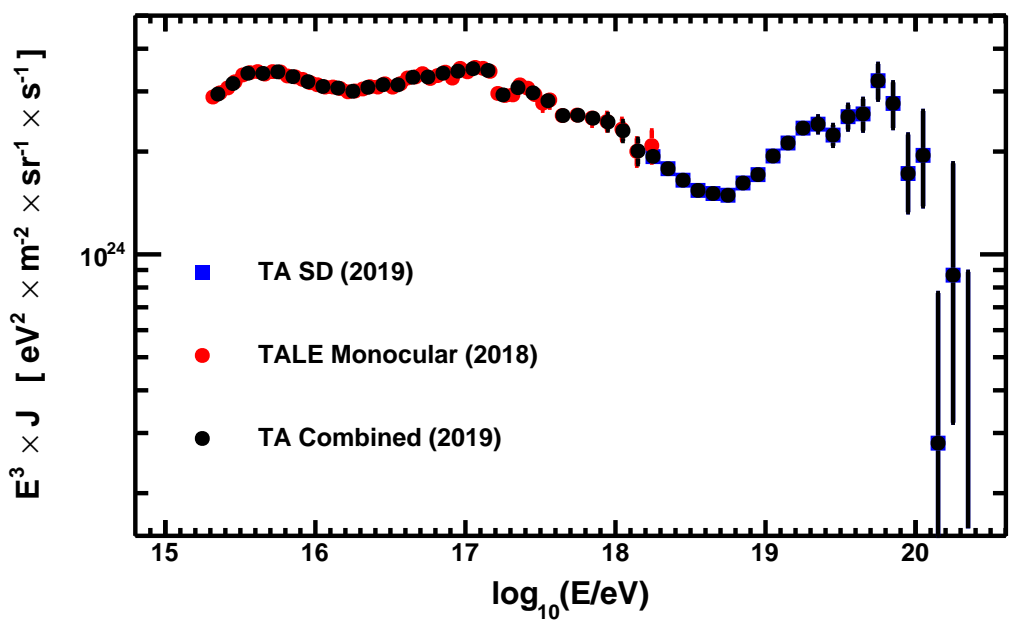

Figure 2: The Telescope Array (TA) energy spectrum (black points) composed of 11 years of the main TA surface detector (SD) data (blue squares) and 22 months of TA Low Energy Extension (TALE) monocular data (red circles). The combined TA spectrum exhibits the knee feature at about $10^{15.5} \mathrm{eV}$, the low energy ankle feature at $10^{16.22 \pm 0.02} \mathrm{eV}$, the second knee at $10^{17.04 \pm 0.04} \mathrm{eV}$, the ankle at $10^{18.69 \pm 0.01} \mathrm{eV}$, and the cutoff at $10^{19.81 \pm 0.03} \mathrm{eV}$.

A new feature, which we call here the shoulder, was initially seen by the Pierre Auger Observatory (Auger) in 2020 [8]. Auger field of view is concentrated mostly in the Southern Hemisphere. It is now interesting to ask whether this new feature also exists in the Northern Hemisphere.

Following the Auger report [8], we have conducted a search for the new shoulder feature in the Northern Hemisphere by combining HiRes I monocular [4], TA Black Rock - Long Ridge monocular [6], and TA SD [7] spectrum measurements to obtain the best statistical power for the Northern Hemisphere. All HiRes and TA detectors employ the same spectrum analysis technique that consists of using detailed Monte Carlo simulations in aperture calculations. To ensure statistical independence of the three measurements, we have additionally removed the TA Black Rock - Long Ridge observation period from the TA SD 11 year spectrum measurement.

Figure 3 shows a joint fit of HiRes I, TA Black Rock - Long Ridge, and TA surface detector spectra into a thrice broken power law. The fit finds the shoulder feature at $10^{19.25 \pm 0.03} \mathrm{eV}$. The statistical significance of the feature is estimated by the procedure described in Figure 3 to be 5.3 standard deviations.

\section{Conclusion}

We conclude that the shoulder feature of the cosmic ray spectrum has been also observed in the Northern Hemisphere by the High Resolution Fly's Eye and the Telescope Array experiments. 


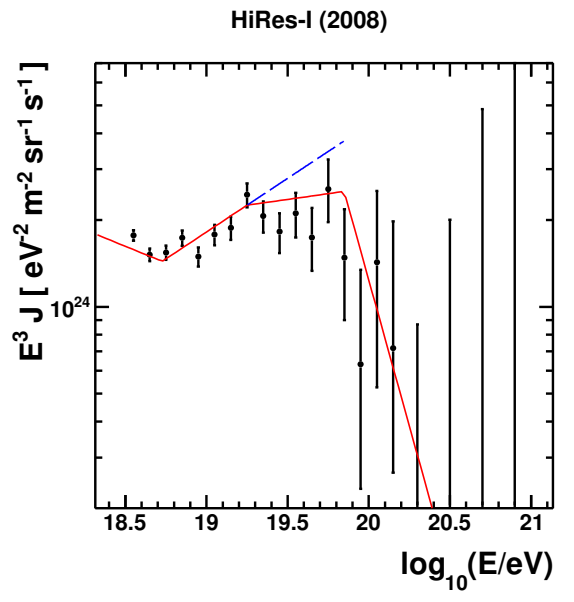

(a)

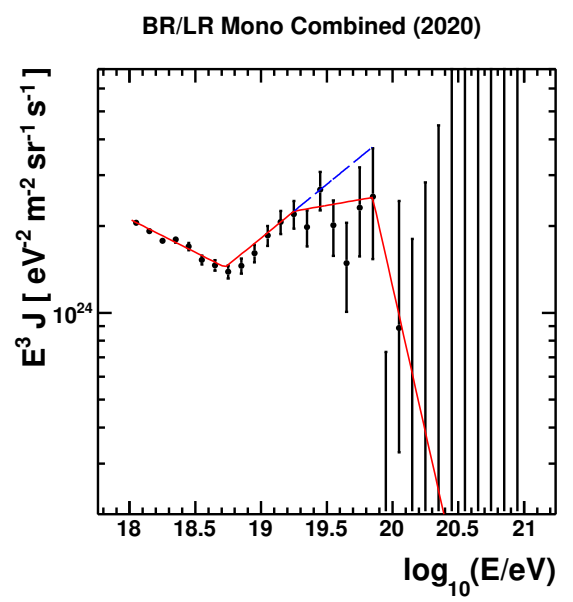

(b)

TA SD (2019) outside of BR / LR Obs. Period

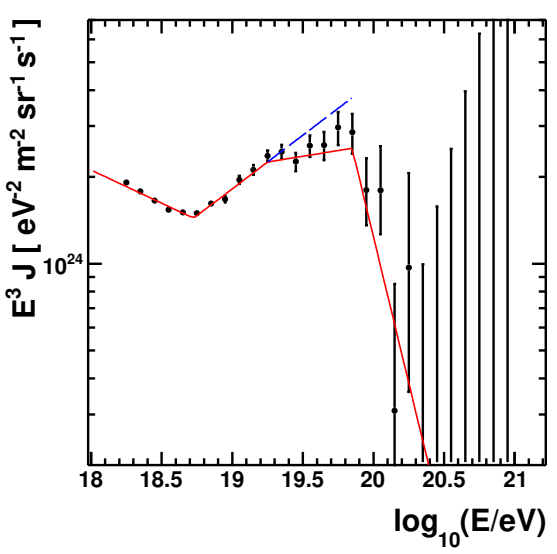

(c)

Figure 3: Joint fit (red line) to the HiRes and TA spectra, which are measured mostly in the Northern Hemisphere. The spectrum shoulder feature is seen at $10^{19.25} \mathrm{eV}$ in (a) HiRes I monocular, (b) TA Black Rock (BR) - Long Ridge (LR) monocular, and (c) TA surface detector (SD) spectra. The significance of the shoulder is found by comparing the number of events expected in the absence of the feature (blue line) and the number of events reported by the experiments. The number of events between $10^{19.25}$ and $10^{19.85} \mathrm{eV}$, expected in the absence of the shoulder, is 1269.3, and the number of events measured by HiRes and TA in that energy range is 1086 . The chance probability of this being a Poisson fluctuation is $7.4 \times 10^{-8}$, or, equivalently, 5.3 standard deviations. 


\section{References}

[1] T. Abu-Zayyad et al., Nucl. Instrum. Meth. A 689 (2012) 87

[2] T. Abu-Zayyad et al., Nucl. Instrum. Meth. A 609 (2009) 227

[3] T. Abu-Zayyad et al., Astropart. Phys. 39-40 (2012) 109

[4] R. U. Abbasi et al., Phys. Rev. Lett. A 100 (2008) 101101

[5] R. U. Abbasi et al. [Telescope Array Collaboration] Astrophys. J. 865, 74 (2018)

[6] D. R. Bergman et al., This conference 2019, 800 (2021).

[7] D. Ivanov, for the Telescope Array Collaboration, PoS ICRC 2019, 298 (2019).

[8] A. Aab et al. [Pierre Auger], Phys. Rev. Lett. 125 (2020) no.12, 121106 


\section{Full author list: The Telescope Array collaboration}

R.U. Abbasi ${ }^{1}$, M. Abe ${ }^{2}$, T. Abu-Zayyad ${ }^{1,3}$, M. Allen ${ }^{3}$, Y. Arai ${ }^{4}$, E. Barcikowski ${ }^{3}$, J.W. Belz ${ }^{3}$, D.R. Bergman ${ }^{3}$, S.A. Blake ${ }^{3}$, I. Buckland ${ }^{3}$, R. $\mathrm{Cady}^{3}$, B.G. Cheon ${ }^{5}$, J. Chiba ${ }^{6}$, M. Chikawa ${ }^{7}$, T. Fujii ${ }^{8}$, K. Fujisue ${ }^{7}$, K. Fujita ${ }^{4}$, R. Fujiwara ${ }^{4}$, M. Fukushima ${ }^{7,9}$, R. Fukushima ${ }^{4}$, G. Furlich ${ }^{3}$, R. Gonzalez ${ }^{3}$, W. Hanlon ${ }^{3}$, M. Hayashi ${ }^{10}$, N. Hayashida ${ }^{11}$, K. Hibino ${ }^{11}$, R. Higuchi ${ }^{7}$, K. Honda ${ }^{12}$, D. Ikeda ${ }^{11}$, T. Inadomi ${ }^{13}$, N. Inoue ${ }^{2}$, T. Ishii ${ }^{12}$, H. Ito ${ }^{14}$, D. Ivanov ${ }^{3}$, H. Iwakura ${ }^{13}$, H.M. Jeong ${ }^{15}$, S. Jeong ${ }^{15}$, C.C.H. Jui ${ }^{3}$, K. Kadota ${ }^{16}$, F. Kakimoto ${ }^{11}$, O. Kalashev ${ }^{17}$, K. Kasahara ${ }^{18}$, S. Kasami ${ }^{19}$, H. Kawai ${ }^{20}$, S. Kawakami ${ }^{4}$, S. Kawana ${ }^{2}$, K. Kawata ${ }^{7}$, E. Kido ${ }^{14}$, H.B. Kim ${ }^{5}$, J.H. Kim ${ }^{3}$, J.H. Kim ${ }^{3}$, M.H. Kim ${ }^{15}$, S.W. Kim ${ }^{15}$, Y. Kimura ${ }^{4}$, S. Kishigami ${ }^{4}$, Y. Kubota ${ }^{13}$, S. Kurisu ${ }^{13}$, V. Kuzmin ${ }^{17 *}$, M. Kuznetsov ${ }^{17,21}$, Y.J. Kwon ${ }^{22}$, K.H. Lee ${ }^{15}$, B. Lubsandorzhiev ${ }^{17}$, J.P. Lundquist ${ }^{3,23}$, K. Machida ${ }^{12}$, H. Matsumiya ${ }^{4}$, T. Matsuyama ${ }^{4}$, J.N. Matthews ${ }^{3}$, R. Mayta ${ }^{4}$, M. Minamino ${ }^{4}$, K. Mukai ${ }^{12}$, I. Myers ${ }^{3}$,

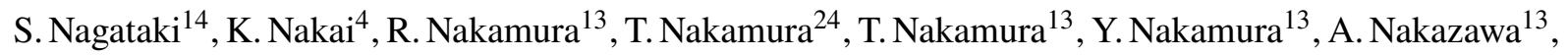
T. Nonaka ${ }^{7}$, H. Oda ${ }^{4}$, S. Ogio ${ }^{4,25}$, M. Ohnishi ${ }^{7}$, H. Ohoka ${ }^{7}$, Y. Oku ${ }^{19}$, T. Okuda ${ }^{26}$, Y. Omura ${ }^{4}$, M. Ono ${ }^{14}$, R. Onogi ${ }^{4}$, A. Oshima ${ }^{4}$, S. Ozawa ${ }^{27}$, I.H. Park ${ }^{15}$, M. Potts ${ }^{3}$, M.S. Pshirkov ${ }^{17,28}$, J. Remington ${ }^{3}$, D.C. Rodriguez ${ }^{3}$, G.I. Rubtsov ${ }^{17}$, D. Ryu ${ }^{29}$, H. Sagawa ${ }^{7}$, R. Sahara ${ }^{4}$, Y. Saito ${ }^{13}$, N. Sakaki ${ }^{7}$, T. $\mathrm{Sako}^{7}$, N. Sakurai ${ }^{4}$, K. Sano ${ }^{13}$, K. Sato ${ }^{4}$, T. Seki ${ }^{13}$, K. Sekino ${ }^{7}$, P.D. $\mathrm{Shah}^{3}$, Y. Shibasaki ${ }^{13}$, F. Shibata ${ }^{12}$, N. Shibata ${ }^{19}$, T. Shibata ${ }^{7}$, H. Shimodaira ${ }^{7}$, B.K. Shinn ${ }^{29}$, H.S. Shin ${ }^{7}$, D. Shinto ${ }^{19}$, J.D. Smith ${ }^{3}$, P. Sokolsky ${ }^{3}$, N. Sone ${ }^{13}$, B.T. Stokes ${ }^{3}$, T.A. Stroman ${ }^{3}$, T. Suzawa ${ }^{2}$,

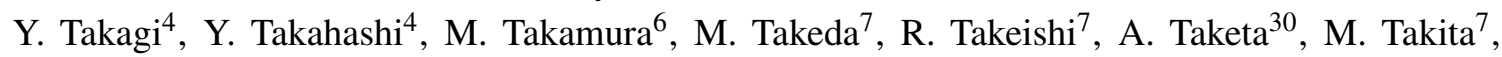
Y. Tameda ${ }^{19}$, H. Tanaka ${ }^{4}$, K. Tanaka ${ }^{31}$, M. Tanaka ${ }^{32}$, Y. Tanoue ${ }^{4}$, S.B. Thomas ${ }^{3}$, G.B. Thomson ${ }^{3}$,

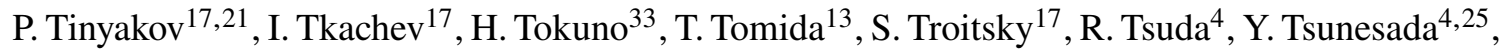

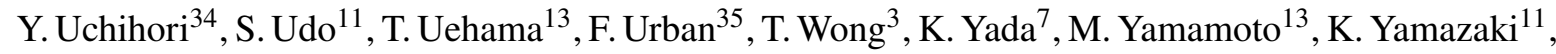

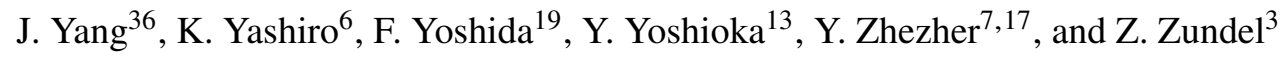

${ }^{1}$ Department of Physics, Loyola University Chicago, Chicago, Illinois, USA

${ }^{2}$ The Graduate School of Science and Engineering, Saitama University, Saitama, Saitama, Japan

${ }^{3}$ High Energy Astrophysics Institute and Department of Physics and Astronomy, University of Utah, Salt Lake City, Utah, USA

${ }^{4}$ Graduate School of Science, Osaka City University, Osaka, Osaka, Japan

5 Department of Physics and The Research Institute of Natural Science, Hanyang University, Seongdong-gu, Seoul, Korea

${ }^{6}$ Department of Physics, Tokyo University of Science, Noda, Chiba, Japan

${ }^{7}$ Institute for Cosmic Ray Research, University of Tokyo, Kashiwa, Chiba, Japan

8 The Hakubi Center for Advanced Research and Graduate School of Science, Kyoto University, KitashirakawaOiwakecho, Sakyo-ku, Kyoto, Japan

${ }^{9}$ Kavli Institute for the Physics and Mathematics of the Universe (WPI), Todai Institutes for Advanced Study, University of Tokyo, Kashiwa, Chiba, Japan

${ }^{10}$ Information Engineering Graduate School of Science and Technology, Shinshu University, Nagano, Nagano, Japan

${ }^{11}$ Faculty of Engineering, Kanagawa University, Yokohama, Kanagawa, Japan

${ }^{12}$ Interdisciplinary Graduate School of Medicine and Engineering, University of Yamanashi, Kofu, Yamanashi, Japan

${ }^{13}$ Academic Assembly School of Science and Technology Institute of Engineering, Shinshu University, Nagano, Nagano, Japan

${ }^{14}$ Astrophysical Big Bang Laboratory, RIKEN, Wako, Saitama, Japan

15 Department of Physics, Sungkyunkwan University, Jang-an-gu, Suwon, Korea

16 Department of Physics, Tokyo City University, Setagaya-ku, Tokyo, Japan

${ }^{17}$ Institute for Nuclear Research of the Russian Academy of Sciences, Moscow, Russia

${ }^{18}$ Faculty of Systems Engineering and Science, Shibaura Institute of Technology, Minato-ku, Tokyo, Japan 
19 Department of Engineering Science, Faculty of Engineering, Osaka Electro-Communication University, Neyagawashi, Osaka, Japan

${ }^{20}$ Department of Physics, Chiba University, Chiba, Chiba, Japan

${ }^{21}$ Service de Physique Théorique, Université Libre de Bruxelles, Brussels, Belgium

${ }^{22}$ Department of Physics, Yonsei University, Seodaemun-gu, Seoul, Korea

23 Center for Astrophysics and Cosmology, University of Nova Gorica, Nova Gorica, Slovenia

${ }^{24}$ Faculty of Science, Kochi University, Kochi, Kochi, Japan

${ }^{25}$ Nambu Yoichiro Institute of Theoretical and Experimental Physics, Osaka City University, Osaka, Osaka, Japan

${ }^{26}$ Department of Physical Sciences, Ritsumeikan University, Kusatsu, Shiga, Japan

${ }^{27}$ Quantum ICT Advanced Development Center, National Institute for Information and Communications Technology, Koganei, Tokyo, Japan

${ }^{28}$ Sternberg Astronomical Institute, Moscow M.V. Lomonosov State University, Moscow, Russia

${ }^{29}$ Department of Physics, School of Natural Sciences, Ulsan National Institute of Science and Technology, UNIST-gil, Ulsan, Korea

${ }^{30}$ Earthquake Research Institute, University of Tokyo, Bunkyo-ku, Tokyo, Japan

31 Graduate School of Information Sciences, Hiroshima City University, Hiroshima, Hiroshima, Japan

32 Institute of Particle and Nuclear Studies, KEK, Tsukuba, Ibaraki, Japan

${ }^{33}$ Graduate School of Science and Engineering, Tokyo Institute of Technology, Meguro, Tokyo, Japan

${ }^{34}$ Department of Research Planning and Promotion, Quantum Medical Science Directorate, National Institutes for Quantum and Radiological Science and Technology, Chiba, Chiba, Japan

${ }^{35}$ CEICO, Institute of Physics, Czech Academy of Sciences, Prague, Czech Republic

${ }^{36}$ Department of Physics and Institute for the Early Universe, Ewha Womans University, Seodaaemun-gu, Seoul, Korea

${ }^{*}$ Deceased 